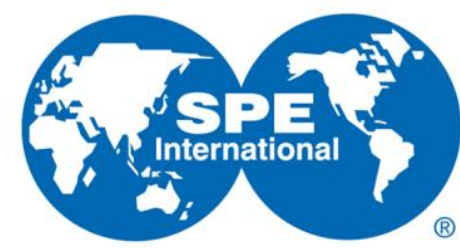

\title{
Detecting Shale Discontinuity between Different Layers Using Waterflood Tomography
}

Yenting Lin, Antonio Ortega, Tayeb Ayatollahy Tafti, SPE and Iraj Ershaghi, SPE

University of Southern California

\section{Abstract}

This paper presents a novel signal processing method to detect the existence and location of shale discontinuities between layers with distinct permeabilities by using only waterflood injection and production data. Continuing the work by Y. Lin, et. al (SPE-132699-PP), we use a new waterflood tomography concept to analyze cross-flow behavior among different layers and estimate the hydraulic transmissivity via tomography mapping. With designed injection rates, we estimate the response times at the producers by monitoring production rates. The response time represents the 'cost' for the fluid to travel between well-pairs and we can use it to model the virtual path through different layers. We consider the case of waterflooding in the presence of permeability contrast in different layers. The fluid in the low permeability layer tends to flow through the shale discontinuity to the higher permeability layer thus forming vertical high permeability cross-flow channels between the wells. With the response time, we are able to identify these 3D high perm channels to estimate the location of shale discontinuities causing the cross-flow. To the best of our knowledge, we are the first to propose this waterflood tomography method. Without significantly affecting daily operation and without the cost associated with tracer testing and seismic tomography, our technique only involves applying scheduled changes to the injection rates and analyzing the relationship between these changes and those in production rates.

To demonstrate the application, using a commercial simulator, we build a double layer reservoir model with different permeabilities. We test a line drive case with injectors and producers having perforations in both lower and upper layers. By applying our designed injection and shut-in schedule, we show our method can provide a very accurate estimate of shale discontinuity location by recording accurate response times between well-pairs.

\section{Introduction}

We consider a geological model to monitor and predict horizontal and vertical sweep in a waterflood project. A practical way to minimize cross flow among layers is to identify and characterize such layers using various mapping techniques. For a reservoir with interlayer cross flow, the earlier depletion occurs in the higher permeability layer. Under this circumstance, we may want to change the injection distributions to improve oil recovery in the lower permeability layer. To achieve this goal, we need to model the cross flow among layers and identify the location of shale discontinuities.

Several different methods have been developed to characterize layered reservoirs. With enough sensors installed, seismic crosshole tomography (Chapman and Pratt, 1992) can provide very high resolution results. 
Tracer testing (Vasco et al. 1999) is also widely used to investigate the flow communication among layers in a reservoir.

Both of these methods, however, require additional cost and may interrupt daily operations. Seismic crosshole testing usually requires significant processing times and has limited to convert the wave velocity to characterize the fluid flow directly. Tracer tests can map the inter-well flow property conveniently, but it is very difficult to repeat the testing in the same area. Lin et al. 2010, use a novel injection schedule as an active input to the reservoir, and measure the changes in production as an output and use the well-pair response time to estimate the high permeability channels. We extend this method to the 3D case in order to identify interlayer shale discontinuities which cause cross layer flow. This method only uses the injection/production data and has the benefit that we can do the measuring without affecting daily operations.

In this paper, we focus on identifying shale discontinuities in a layered reservoir with a large permeability contrast among layers. Because the shale discontinuities provide channels for the fluid to flow between different layers, changes in injection should enable us to observe changes in productions in different layers. The response time for the wells located in different layers then allows us to determine the location where the cross-layer flow happens. To estimate the well-pair response time in a 3D layered reservoir, we improve the injection schedule design and include the option of shutting-in some producers in order to improve observation of the cross flow among layers.

With the injection/production data, we use the capacitance model (CM) (Yousef et al. 2005) to characterize the flow property for each well-pair. This can be viewed as a virtual streamline approach to explain the production according to the interaction between well-pairs. In CM, a 'time delay' constant is introduced to characterize the delay effect of the injection signal at the producers. This 'time delay' constant is associated with the cross layer travel path, which is determined by the location of shale discontinuity.

After retrieving the time delay constant, we treat the injectors and producers as the transmitters and receivers, respectively, and use the time delay constant as the traveltime. The problem of finding the shale discontinuities can then be modeled as a crosshole travel time tomography problem. One special property of this problem is that shale discontinuities may have very different flow properties. This leads to a high contrast travel time tomography problem, which has been studied by Lin and Ortega (2010). Based on their work, we investigate how the shale discontinuity will affect the flow path in a layered reservoir, and improve the reconstruction algorithm.

In what follows, we first define a model for the injection/production response in a heterogeneous reservoir. The connection between flow path and location of shale discontinuity is also explained. Then we introduce the concept of travel time tomography and provide details of the reconstruction algorithm. Finally, we provide simulation results and conclude our work.

\section{Physical Model}

The recently introduced CM provides a powerful tool to evaluate the waterflood performance by treating the injection/production as the system input/output and using signal processing tools to estimate the unknown system parameters. Yousef et al. (2005) showed how to extract reservoir properties based on the parameters of capacitance model.

The mathematical formulation for CM can be written as

$$
\tau \frac{d q}{d t}+q(t)=i(t)-\tau * J \frac{d p_{w f}}{d t},
$$

where $i(t)$ is the injection rate and $q(t)$ is the total production rate. $P_{w f}$ represents the flowing bottom hole pressure (BHP) and $J$ stands for the productivity index. If we consider the case where the reservoir is mature and the bottom-hole pressure is fixed, then we can approximate the $j-t h$ well production rates based on only the contribution from injectors by: 


$$
q_{j}(t)=\sum_{i} \int_{t_{0}}^{t} \frac{e^{-(t-\varsigma) / \tau_{i j}}}{\tau_{i j}} I_{i}(\varsigma) d \varsigma
$$

The 'time constant' $\tau_{i j}$ for each injector-producer pair is defined by the total compressibility $c_{t}$, the productivity index, $J$, and the pore volume $V_{p}$, which are associated with the control volume between injector $i$ and producer $j$ pair.

$$
\tau_{i j}=\left(\frac{c_{t} V_{p}}{J}\right)_{i j}
$$

From the above, it is clear that if we have an area with higher productivity index $J$, the time delay constant will be smaller. This implies that if the time delay constant for a specific well-pair is very small, it is highly likely that most of the corresponding flow path passes through the high permeability layer.

\section{Flow path for high contrast layered reservoir}

For a homogeneous reservoir, the control volume is roughly proportional to the distance between the injector and producer. When we look at the cross-layer flow, the total control volume can be modeled as the cascade of the control volumes in different layers. From the Fermat principle, the flow path will be the path with least cost time and this implies the flow will take advantage of the higher permeability layer because it has less 'resistance' (See Figure 1)

In $\mathrm{CM}$, the time-delay constant is determined by the control volume and is inversely proportional to the productivity index. Because the control volume can be viewed as cascading of the volumes in different layers, the time delay constant will also be the summation of several parts: the time delay constant in upper layer and the one in lower layer. These delays depend on the length of flow paths and the productivities in different layers. Note that the length of flow paths in each layer is determined by the location of shale discontinuities, as these corresponding "entry points" where fluid can travel from one layer to another.

For example, if we assume the productivity index of the low permeability layer is 1 , while that of the higher permeability layer is 10 , the time delay constant can be separated as two segments belonging to different layers

Time delay constant $\sim L_{1} / 1+L_{2} / 10$, with $L=\left\{L_{1} \cup L_{2}\right\}$

$L$ represents the flow path between the injector-producer pair, and the ratio of two segments is controlled by the location of shale discontinuities.
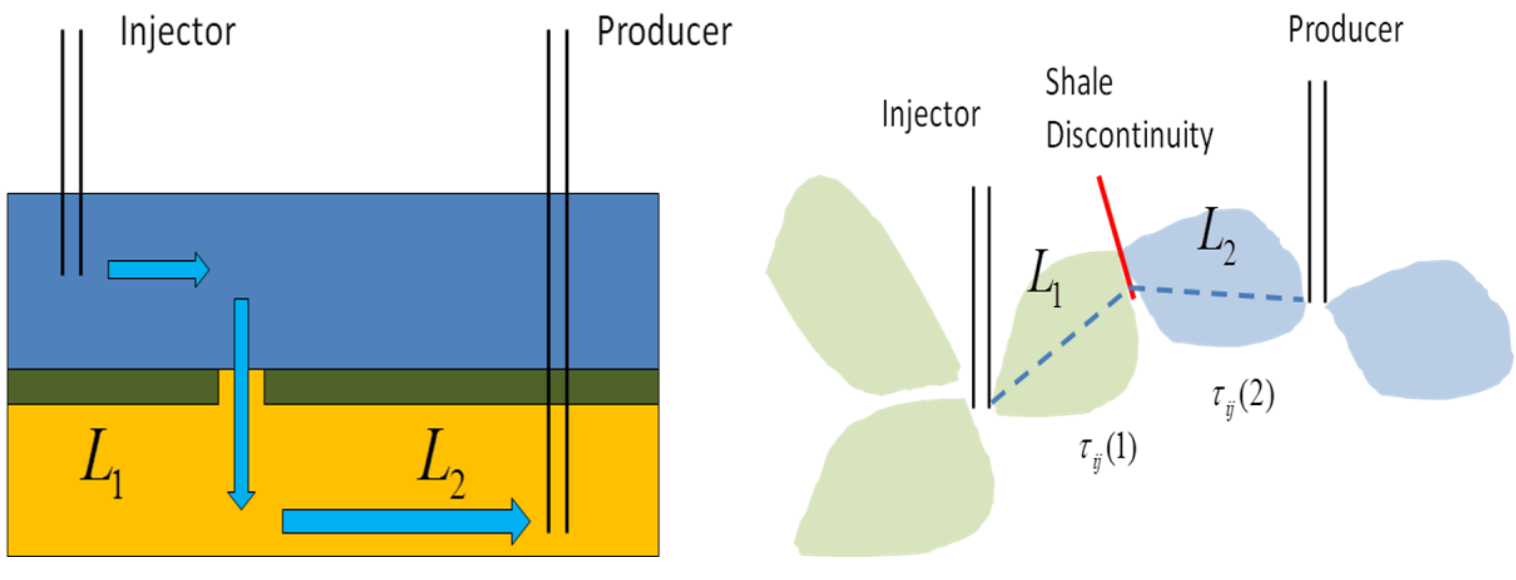
Figure 1: Example of the cross-layer flow. The total control volume is determined by the location of shale discontinuities. (a) The flow path (b) The time delay constant is the sum of time delay constants in different layers.

\section{Injection schedule design}

In order to get a reliable estimate of the parameters for CM, we use the well-known Pseudo-Noise (PN) sequence as the injection schedule. By using PN sequence as the input, we can achieve estimates of FIR model parameters with the lowest error covariance. (Ljung, 1987) This procedure has been proposed for reservoir modeling by Lee et al. (2008). Here we extend it by including the option of shutting-in some of the producers.

When we change the injection rate in the lower permeability layer, usually we can detect production changes in all layers. However, it is very hard to observe production changes caused by the cross-layer flow when we injection rates are changed only in the high permeability layers. This is because high permeability layers provide a low 'resistance' pathway for the fluid, so that most of the fluid will stay in these layers and only a small amount will flow through shale discontinuities to the low permeability layers. Therefore, in designing our injection/production schedule we allow producers to be shut-in in order to divert the fluid flow to a different layer. For example, if a producer obtaining fluid from a high permeability layer is shut-in, this will make it easier to observe the response of a producer corresponding to a low permeability layer.

The whole schedule can be viewed as an alternating process: We apply PN sequences as injection schedule for wells in one specific layer, and while shutting down production in the same layer. If we observe a change of production this implies the existence of shale discontinuities that cause cross-layer flow.

\section{Reconstruction Algorithm}

The problem of identifying shale discontinuities with cross-layer time delay constant can be seen as a high contrast travel time tomographic reconstruction, which has been studied by Lin and Ortega (2010). One of the major difficulties for reconstruction algorithm design is that the sensor locations are spatially sparse, i.e., limited to the locations of injectors/producers, while the flow velocity contrast between the layers is very high. If we use a cell based inversion (Justice et al. 1989) method, which divided the volume into small cells, the result will be a very blurred image, where it will be hard to identify the shale discontinuities.

Instead of using a cell-based method, we choose an object-based representation for the shale discontinuities. We describe the geometrical shape of the shale discontinuities using the pre-defined objects, which require much fewer parameters to be estimated, as compared to the cell-based methods. Our algorithm, based on a forwardbackward procedure, iteratively modifies the object parameters in order to match the measured delay time constant.

In the forward step, we calculate the time delay constant based on the current model parameters. If the time delays fit the measurements, then we claim this is a valid solution and stop the algorithm. Otherwise, we go to the backward step to update the locations of shale discontinuities based on the difference between the current predicted time delay constants and those obtained from the measurements. This is summarized in Figure 2. 


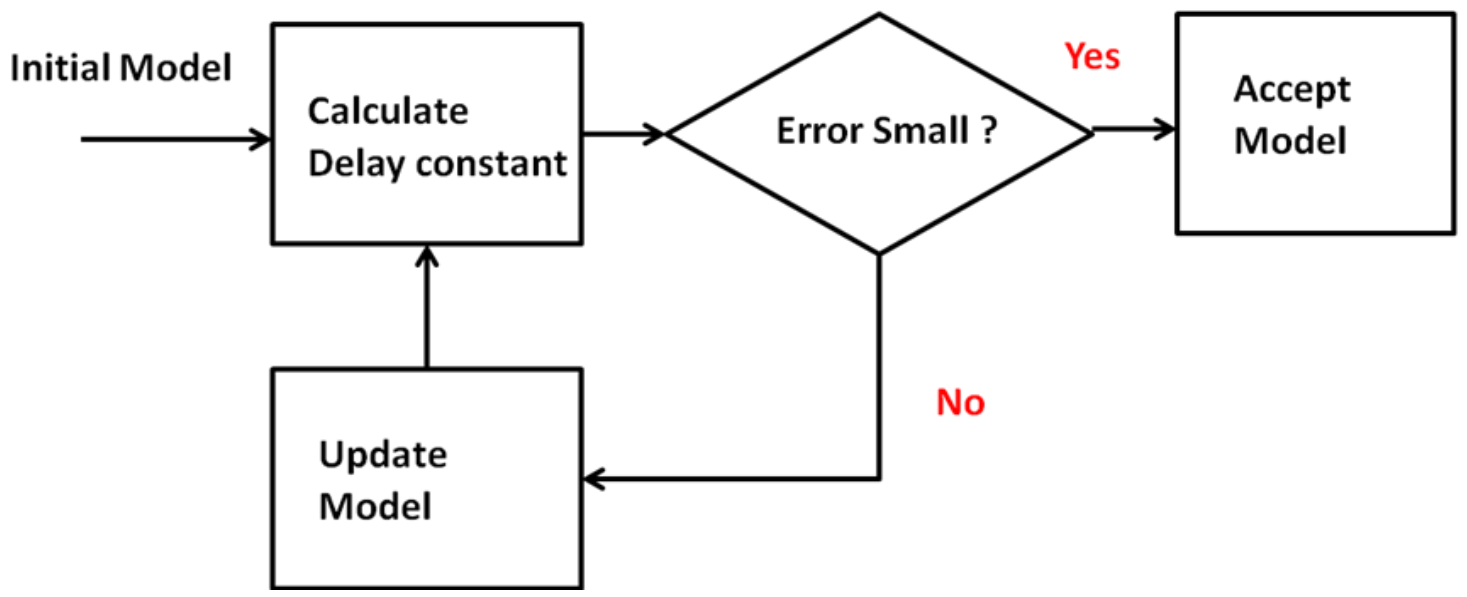

Figure 2: Flow chart of the `forward-backward' procedure

In the 'forward' step, given the current model parameters, we can define the shape and location of shale discontinuities and calculate the flow path. The flow travel path is determined by the location of shale discontinuities, which act as entry points between the different layers (See Figure 1). If the permeability contrast is very high, the flow path can be approximated as a combination of line segments that represent the shortest distance from wells to shale discontinuities. Then, the time delay constant can be estimated to be proportional to the sum of travel distances inside each of the layers.

For example, if the lower layer has much higher permeability than the upper layer, with the current location of shale discontinuity the flow path can be separated into two segments belonging to different layers (See Figure 3). Because the higher permeability layer is less 'resistant', the flow path will seek the earliest possible entrance from the lower permeability layer.

As compared to path_2, path_1 has much shorter travel distance in the low permeability layer, so path_1 will have less high 'resistant' component. As a consequence, path_1 will dominate the control volume and also the time delay constant. Therefore, for a given estimate of the location of shale discontinuities, the travel path can be approximated by the shortest path, in the low permeability layer, between the well and the shale discontinuity.
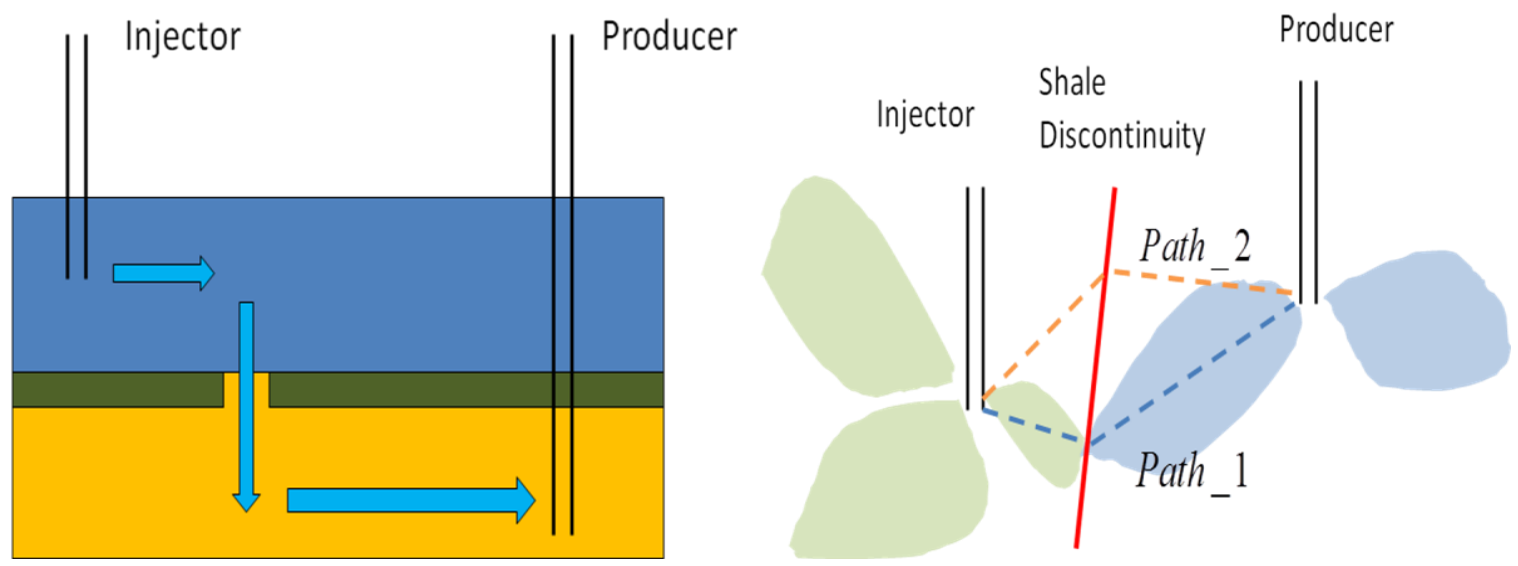

Figure 3: Example of the flow path given the location of shale discontinuities. (a) The wells are located in different layers (b) Compare two possible flow path. Path_1 has shortest distance in low perm layer.

In the 'backward' step, we are trying to update the model parameters based on the mismatch between the measured and the predicted time delay constants. Because the location of shale discontinuities is determined by the object parameters, we can formulate the updating as a parameter estimation problem 


$$
\theta=\underset{\theta}{A \operatorname{rg} \min }\|T(\theta)-t\|
$$

$T(\theta)$ is the predicted time delay constant based on object parameter $\theta$, and $t$ is the measured time delay constant, and so the goal is to identify those parameters that minimize the error. In Figure 4, we depict a two layer reservoir where the shale discontinuities are represented by line structures. When we change the object parameters, e.g., shifting or rotating the location of shale discontinuities, we can use the formula we derive in the forward step to predict the change in the time delay constant. Then we use a gradient search algorithm in order to find the optimal parameters, corresponding to shale discontinuity locations that minimize the mismatch.

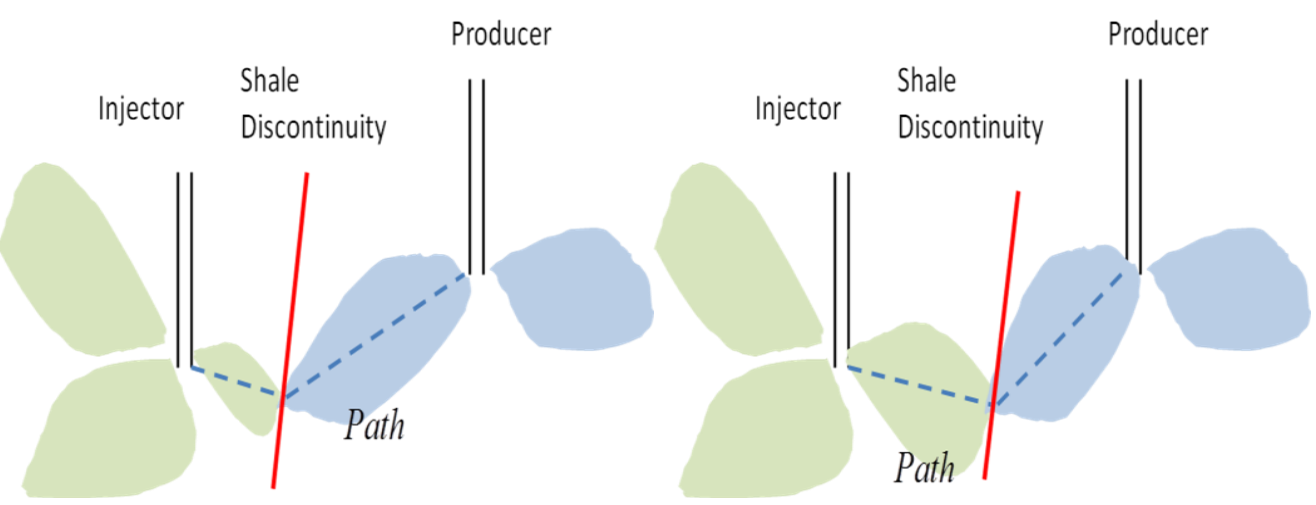

Figure 4: Example of the change in flow path w.r.t the model parameters. We can predict the change in flow path if we shift, or rotate the location of shale discontinuities.

\section{Simulation Results}

We build a two layer reservoir with high permeability contrast and use a commercial simulator to test our method. The testing case is a line drive with 5 injectors and 5 producers. 3 of the injectors/producers are in the low permeability layers, and others are in the high permeability layers. We put a simple shale discontinuity and assign high permeability for it to simulate the effect.

We use the PN sequence as the injection schedule and shut-in the producers in the same layer for the testing period. The changes of production can be measured for the producers located in different layers. We retrieve the time delay constant in CM by injection/production data, and use it to identify the location of shale discontinuities. The result shows our method can estimate the location very accurately.

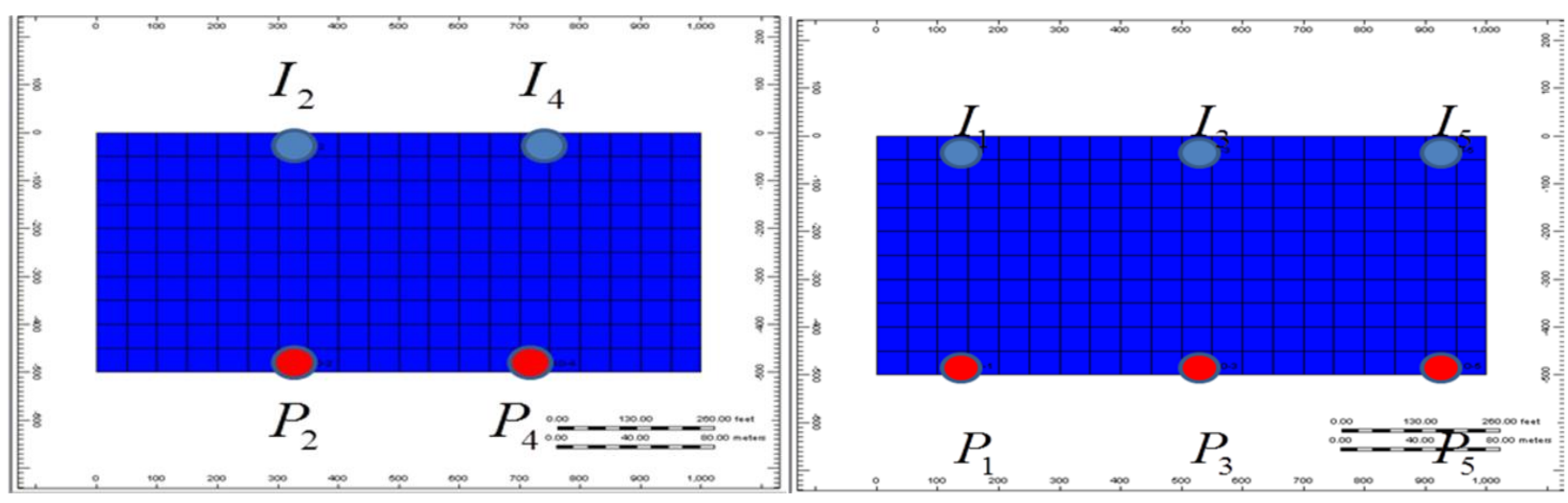




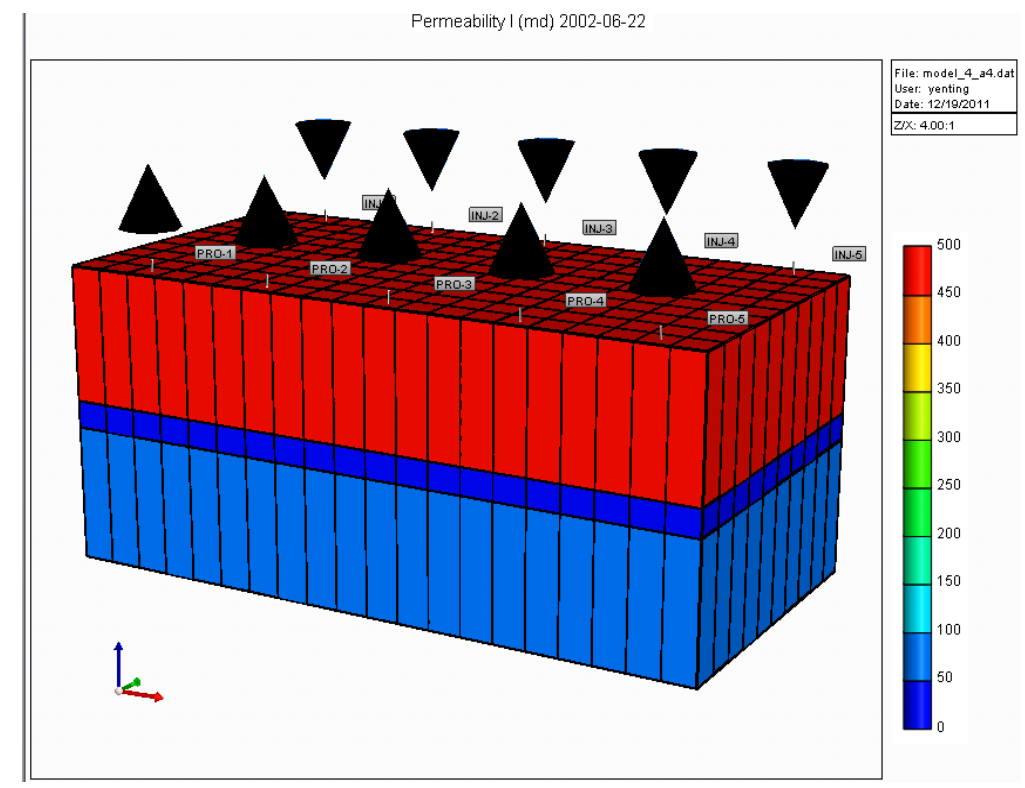

Figure 5: Testing scenario in the simulator: line drive, two layer reservoir with 5 injectors/producers. 3 are in the low perm layer and 2 are in high perm layer. (a) 2D view of upper layer (b) 2D view of lower layer (c) 3D view
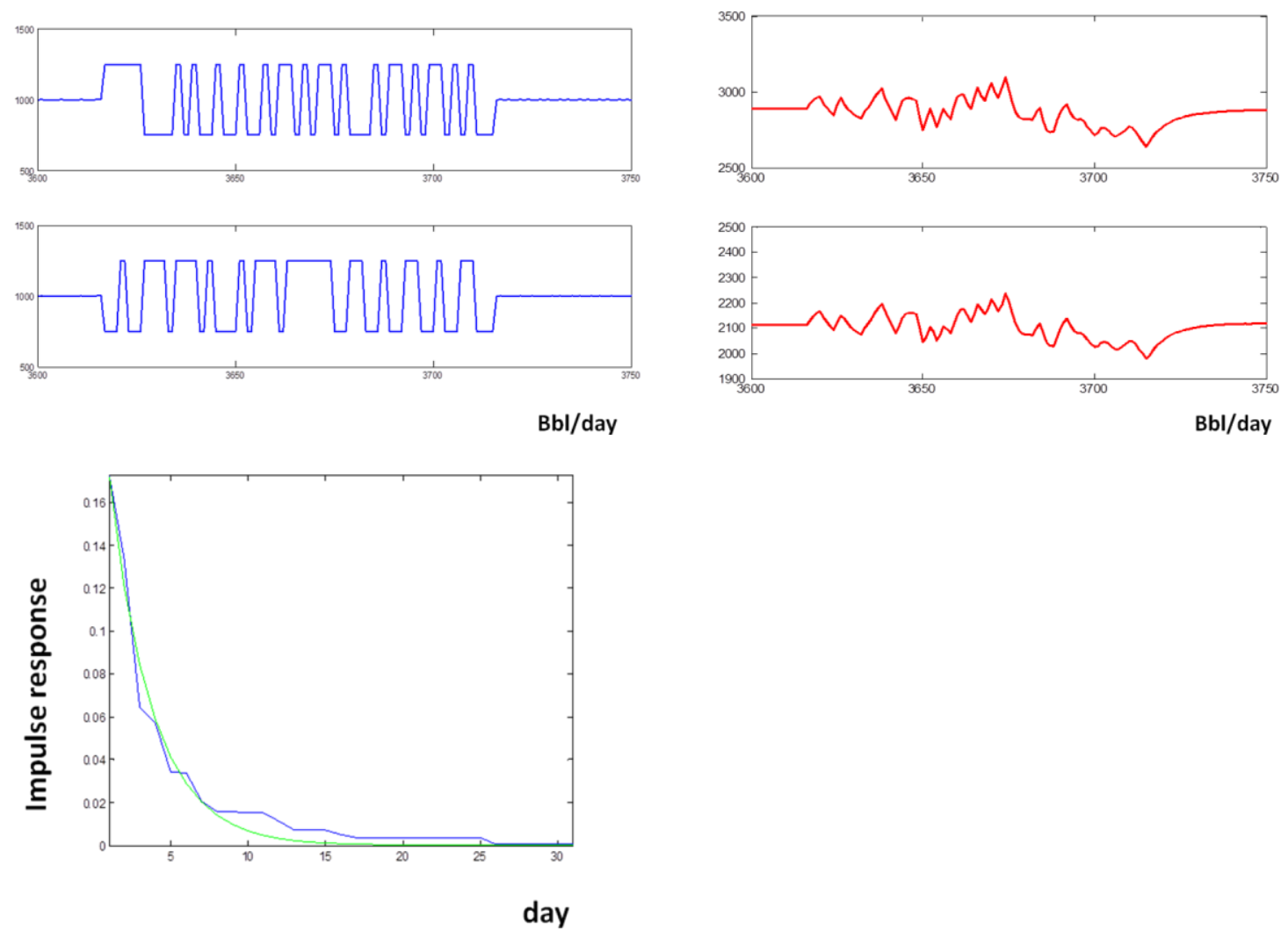

Figure 6: (a) The designed injection schedule. (b) The measured production rate. (c) The estimated FIR and we match it with $\mathrm{CM}$ to retrieve the time delay constant. 

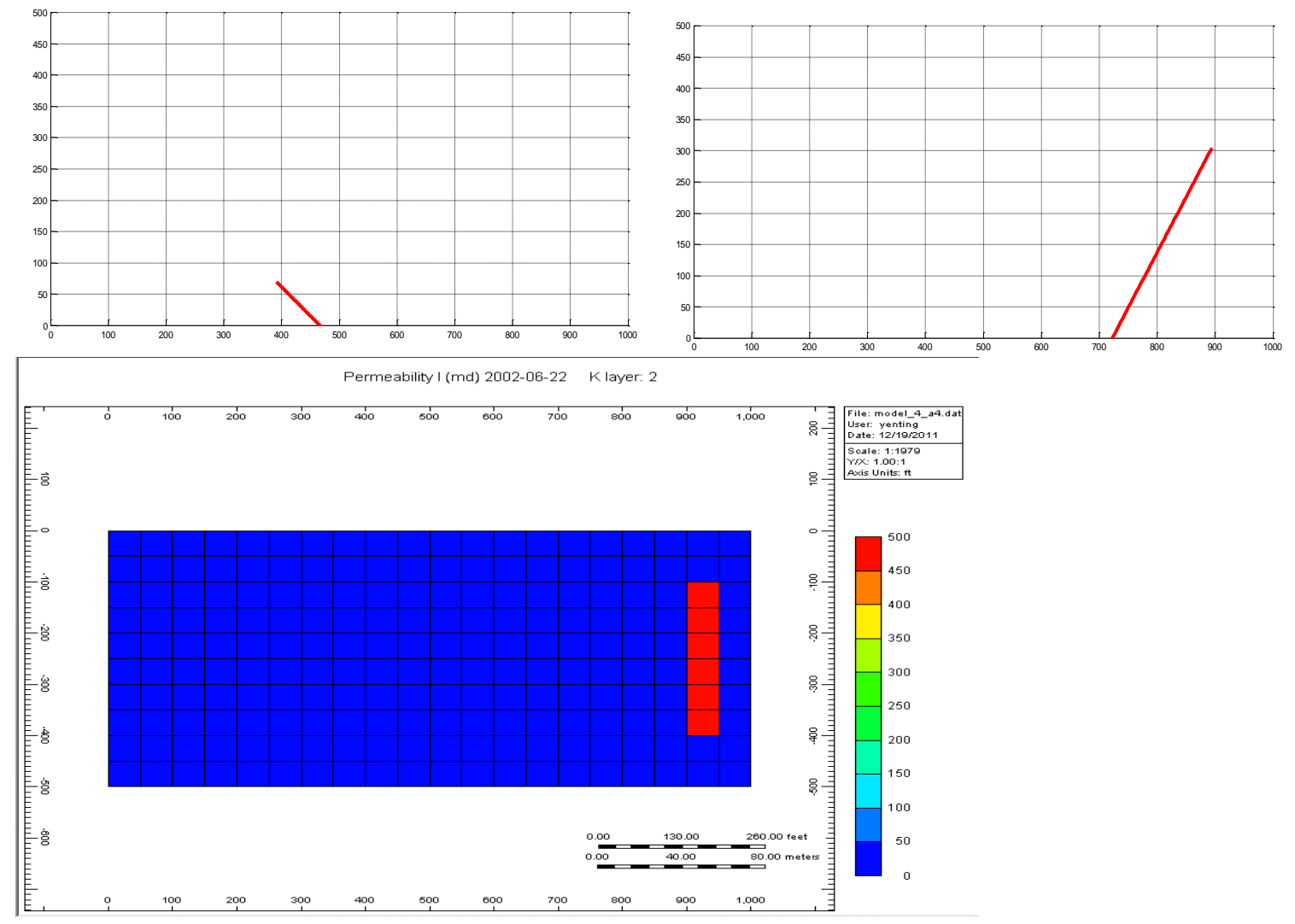

Figure 7: Identification result of the shale discontinuity. (a) Initial structure. (b) The output of our algorithm (c) The ground truth in simulator.

\section{Conclusion}

In this paper, we propose a new method to identify the shale discontinuities by using the water injection as an active input source and measuring the cross-layer change in production. This method only needs the injection/production data and does not require any additional instruments.

To apply our method in a real field, one of the critical issues is the sampling frequency and accuracy of the data. Usually we have very reliable and frequent injection data, but production rates are obtained from the welltests, which are performed on a weekly basis. It limits the time-resolution when we try to retrieve the time delay constant. For example, assume the time delay constants for the well-pairs are 1 and 4 days, when we increase the injection rates we will see almost the same changes in weekly production rate. To deal with this problem, we suggest using the Infer Production provided by pump of controller which provides more frequent production data.

Future work will be focusing on how to improve the reconstruction algorithm by considering the geological prior information. Also, we plan to do a field test with our designed injection schedule.

\section{Acknowledgements}

This work is supported in part by Chevron Corp. under the joint project Center for Interactive Smart Oilfield Technologies (CiSoft), at the University of Southern California.

\section{Reference}


Vasco, D.W., Yoon, S. and Datta-Gupta, A. "Integrating dynamic data into high-resolution reservoir models using streamline-based analytic sensitivity coefficients,” SPE Journal, vol. 4, no. 4, 1999.

Yousef, A.A, Gentil, P., Jensen, J. and Lake, L. "A capacitance model to infer interwell connectivity from production and injection rate fluctuations," in SPE Annual Technical Conference and Exhibition, 2005.

Berryman, J.G., "Lecture notes on Nonlinear Inversion and Tomography: I. Borehole seismic tomography." Tech. Rep., UCRL-LR-105358-Rev. 1, Lawrence Livermore National Lab., CA (United States), 1991.

Lin, Y.T. and Ortega, A., (2010) "Reconstruction algorithm for high contrast velocity travel time tomography," in Proc. ICASSP, Dallas, TX, USA, Mar. 2010.

Johnson, C.R., Greenkorn, R.A. and Woods, E.G. "Pulse testing: a new method for describing reservoir flow properties between wells," Journal of Petroleum Technology, vol. 18, no. 12, pp. 1599-1604, 1966.

Lee, K.H., Ortega, A., Ghareloo, A. and Ershaghi, I. "A Method for Characterization of Flow Units between Injection-Production Wells Using Performance Data," in SPE Western Regional and Pacific Section AAPG Joint Meeting, 2008.

Chapman, $\mathrm{CH}$ and Pratt, RG. "Traveltime tomography in anisotropic media-I. Theory," Geophysical Journal International, vol. 109, no. 1, pp.1-19, 1992.

Ljung, L. "System identification: theory for the user. 1987," PTR Prentice Hall, Englewood Cliffs, New Jersey.

J. H. Justice, A. A. Vassiliou, S. Singh, J. D. Logel, P. A. Hansen, B. R. Hall, P. R. Hutt, and J. J. Solanki "Acoustic tomography for monitoring enhanced oil recovery," Geophysics: The Leading Edge, February 1989, v.8, p.12-19 